Chapter 21

\title{
The Role of Carbon Nanotubes in Enhancement of Photocatalysis
}

Tawfik A. Saleh

Additional information is available at the end of the chapter

http://dx.doi.org/10.5772/51050

\section{Introduction}

The chemical, physical and mechanical properties of carbon nanotubes (CNTs) have stimulated extensive investigation since their discovery in the early 1990s (Iijima, 1991). CNTs, which are considered quasi-one dimensional nanostructures, are graphite sheets rolled up into cylinders with diameters of the order of a few nanometers and up to some millimeters in length. Types of nanotubes are the single-walled nanotubes (SWCNTs), double-walled nanotubes (DWCNTs) and the multi-walled nanotubes (MWCNTs). The MWCNTs consist of multiple layers of graphite arranged in concentric cylinders.

During the early stage, the primary research interests include the synthesis or growth of CNTs to prepare enough amounts of CNTs with desired dimension and purity. Several methods like arc discharge, laser ablation of graphite, the more productive chemical vapor deposition (CVD) and plasma enhanced CVD method, have been used to prepare high purity CNTs with controllable wall-thickness and length and acceptable price (Meyyappan, 2004). CNTs attract considerable attention due to their special structure and high mechanical strength which makes them to be good candidates for advanced composites. They can be either semiconducting, semimetallic or metallic, depending on the helicity and the diameter of the tube (Ebbesen et al., 1996; Yang et al., 2003). Based on the structure and shape, CNTs conduct electricity due to delocalization of the pi bond electrons. On the other side, researchers found that CNTs are efficient adsorbents due to their large specific surface area, hollow and layered structures and the presence of pi bond electrons on the surface. Besides that, more active sites can be created on the nanotubes. Thus, CNTs can be used as a promising material in environmental cleaning.

Photocatalytic oxidation using a semiconductor such as $\mathrm{TiO}_{2}, \mathrm{ZnO}$ and $\mathrm{WO}_{3}$ as photocatalyst is one of the advanced oxidation processes used for degradation of various pollutants in in- 
dustrial wastewaters. As the semiconductor is illuminated with photons having energy content equal to or higher than the band gap, the photons excite valence band (VB) electrons across the band gap into the conduction band $(\mathrm{CB})$, leaving holes behind in the valence band.Thus, there must be at least two reactions occurring simultaneously: oxidation from photogenerated holes, and reduction from photogenerated electrons.

The holes react with water molecules or hydroxide ions $\left(\mathrm{OH}^{-}\right)$producing hydroxyl radicals $\left({ }^{\circ} \mathrm{OH}\right)$. The generation of such radicals depends on the $\mathrm{pH}$ of the media. Targeted pollutants which are adsorbed on the surface of the catalyst will then be oxidized by ${ }^{\bullet} \mathrm{OH}$. On the other hand, the excited electrons (e-) to the conduction band (CB) can generate hydroxyl radical $\left({ }^{\circ} \mathrm{OH}\right)$ and can also react with $\mathrm{O}_{2}$ and trigger the formation of very reactive superoxide radical ion $\left(\mathrm{O}_{2}^{-\bullet}\right)$ that can oxidize the target.

The band gap is characteristic for the electronic structure of a semiconductor and is defined as the energy interval $\left(\Delta \mathrm{E}_{\mathrm{g}}\right)$ between the VB and CB (Koci et al.,2011). VB is defined as the highest energy band in which all energy levels are occupied by electrons, whereas $\mathrm{CB}$ is the lowest energy band without electrons. The rate of a photo catalytic reaction depends on several parameters. First and most important is the type of the photo catalytic semiconductor. The second factor is the light radiation used or the stream of photons, as over supply of light accelerates electron-hole recombination (Koci et al.,2008). Third factor is $\mathrm{pH}$ of the medium with which the semiconductor surface is in contact with the targeted molecules. Fourth factor is the concentration of the substrate influencing the reaction kinetics. Fifth parameter is the temperature of the media where higher temperatures cause frequent collision between the semiconductor and the substrate (Koci et al.,2010).

The degradation rate can be enhanced by reducing the electron-hole recombination rate; preventing the particles agglomeration; and increasing the adsorption capacity, as it is a key process in the photocatalysis. In order to improve the photocatalytic efficiency, several methods have been investigated. This includes:

1. increasing the surface area of the metal oxide by synthesizing nano-size materials;

2. generation of defect structures to induce space-charge separation and thus reduce the recombination;

3. modification of the semiconductors with metal or other semiconductor; and

4. adding a co-sorbent such as silica, alumina, zeolite or clay (Yu et al. 2002; Rusu and Yates, 1997)

CNTs based composites have attracted considerable attentions due to the intrinsic properties that have been created owing to the addition of CNTs into the composite. Functionalization of CNTs, or attachment of individual atoms, molecules or their aggregates to CNTs, further extend the field of application of these nanosystems in different fields like in photocatalysis process (Dresselhaus \& Dresselhaus, 2001; Burghard, 2005; Saleh, 2011). CNT/Metal oxide composites have been recently reported to be used for the treatment of contaminated water. In this chapter, therefore, the application of CNTs to enhance the photocatalytic activity of $\mathrm{TiO}_{2}, \mathrm{ZnO}$ and $\mathrm{WO}_{3}$ will be discussed. 


\section{Synthesis of carbon nanotube/catalyst composites}

There are two main steps for the synthesis of CNT/catalyst nanocomposites. The first step is the grafting of oxygen-containing groups on the surface of the nanotubes and the second step is the attachment of the metal oxides on the active surface of the nanotubes.

\subsection{Grafting of oxygen-containing groups on CNTs}

Grafting of oxygen-containing groups on the surface of the nanotubes or activation of CNTs can be achieved by oxidation treatment. It can be performed using oxidizing agents such as nitric acid, sulfuric acid, or a mixture of both. For example, oxygen-containing groups can be grafted on the surface of the nanotubes by the following procedure. Initially, CNTs are dispersed by sonication in concentrated acidic media. Then, the mixture is treated by reflux while stirring vigorously at temperature of $100-120^{\circ} \mathrm{C}$. After refluxing process, the mixture is allowed to cool at room temperature. The oxidized CNTs are purified by extraction from the residual acids by repeated cycles of dilution with distilled water, centrifugation and decanting the solutions until the $\mathrm{pH}$ is approximately 5-6. After the purification process, the oxidized CNTs are dried overnight in an oven at $100^{\circ} \mathrm{C}$. After that, the dry oxidized CNTs are pulverized in a ball-mill.

The presence of oxygen containing groups on the surface of the oxidized nanotubes are characterized by the means of Fourier transform infrared spectroscopy (FT-IR), X-ray powder diffraction (XRD), field emission scanning electron microscopy (FESEM ) and the transmission electron microscopy (TEM).

As an example, IR spectra, in the range of $400-4000 \mathrm{~cm}^{-1}$, were recorded in $\mathrm{KBr}$ pellets using a Thermo Nicolet FT-IR spectrophotometer at room temperature. Samples were prepared by gently mixing $10 \mathrm{mg}$ of each sample with $300 \mathrm{mg}$ of $\mathrm{KBr}$ powder and compressed into discs at a force of $17 \mathrm{kN}$ for 5 min using a manual tablet presser. Figure 1 depicts IR spectrum of oxidized MWCNTs. In the spectrum, a characteristic peak at $1580 \mathrm{~cm}^{-1}$ can be assigned to $\mathrm{C}=\mathrm{C}$ bond in MWCNTs. The band at about $1160 \mathrm{~cm}^{-1}$ is assigned to $\mathrm{C}-\mathrm{C}$ bonds. Also, the spectrum shows the carbonyl characteristic peak at $1650 \mathrm{~cm}^{-1}$, which is assigned to the carbonyl group from quinine or ring structure. More characteristic peak to the carboxylic group is the peak at $1720 \mathrm{~cm}^{-1}$ (Ros et al., 2002; Yang et al., 2005; Xia et al., 2007). The observation of IR spectra corresponding to the oxidized MWCNTs suggests the presence of carboxylic and hydroxylic groups on the nanotube surface.

Figure 2 depicts the typical XRD pattern of the oxidized MWCNTs. The strongest diffraction peak at the angle $(2 \theta)$ of $25.5^{\circ}$ can be indexed as the $C(002)$ reflection of the hexagonal graphite structure (Rosca et al., 2005; Saleh et al., 2011; Lu et al., 2008). The sharpness of the

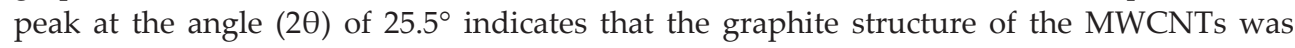
acid-oxidized without significant damage since any decrease in the order of crystallinity in 
MWCNTs will make the XRD peaks broader and shift the peak diffraction towards lower angles. The other characteristic diffraction peaks of graphite at $2 \theta$ of about $43^{\circ}, 53^{\circ}$ and $77^{\circ}$ are associated with $\mathrm{C}(100), \mathrm{C}(004)$ and $\mathrm{C}(110)$ diffractions of graphite, respectively.

Energy dispersive X-ray spectroscope (EDX) measurement is also used as a quantitative analysis for the presence of the oxygen containing groups on the surface of the nanotubes. Figure 4 represents the results of the oxidized MWCNTs. The results shows the presence of oxygen in the sample in addition to carbon element. SEM and TEM are used to characterize the morphology of the nanotube and to ensure that the structure of the nanotube has not been destroyed by the acid treatment. As an example, SEM image and the inset TEM image in Figure 3 confirm that there is no damage effect on the nanotubes using mixtures of nitric acid sulfuric acid for the treatment of the nanotubes.

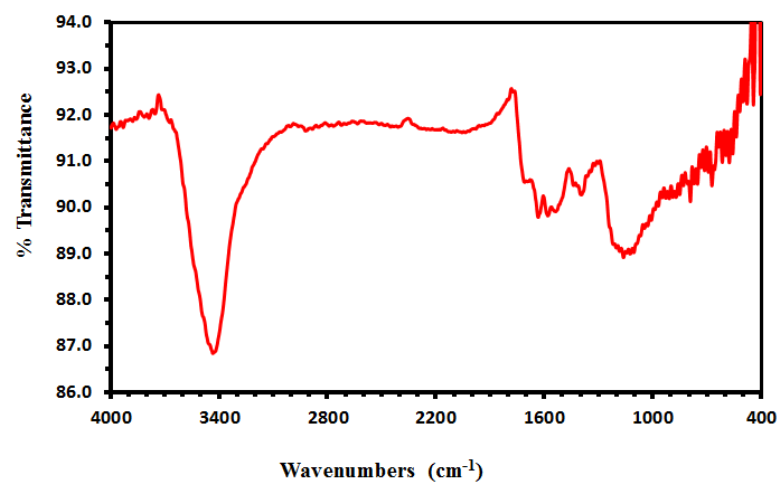

Figure 1. FTIR spectrum of MWCNT oxidized with $\mathrm{H}_{2} \mathrm{SO}_{4} / \mathrm{HNO}_{3}$ mixture for $6 \mathrm{~h}$ at $100^{\circ} \mathrm{C}$.

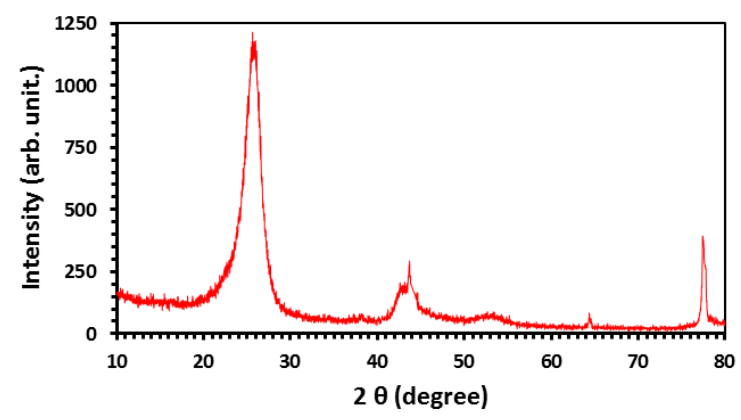

Figure 2. XRD patterns of MWCNT oxidized with $\mathrm{H}_{2} \mathrm{SO}_{4} / \mathrm{HNO}_{3}$ mixture for $6 \mathrm{~h}$ at $100^{\circ} \mathrm{C}$. 


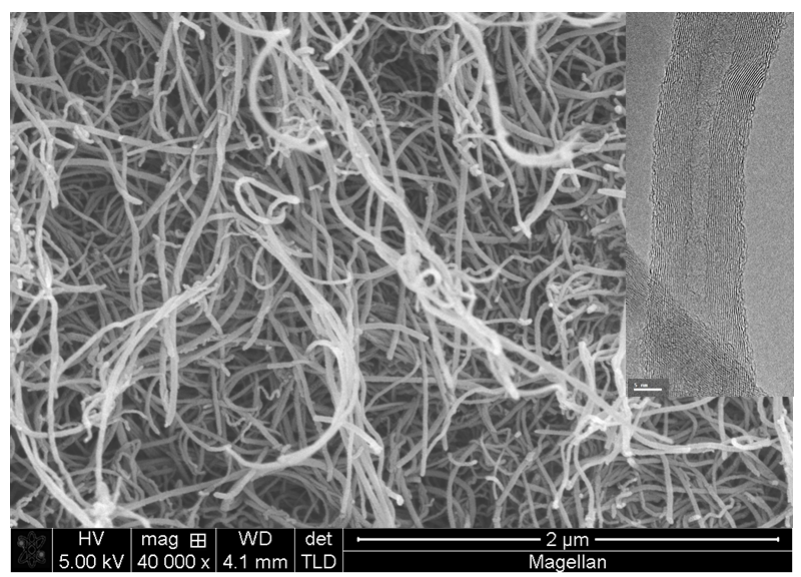

Figure 3. Field emission scanning electron microscopy (FESEM) image of the MWCNTs oxidized with $\mathrm{H}_{2} \mathrm{SO}_{4} / \mathrm{HNO}_{3}$ mixture for $6 \mathrm{~h}$ at $100^{\circ} \mathrm{C}$; Inset is the transmission electron microscopy (TEM) image of the same.

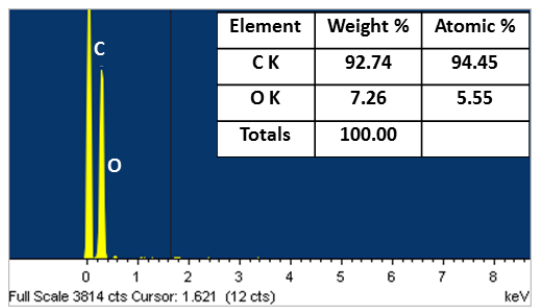

Figure 4. EDX spectrum of the MWCNTs oxidized with $\mathrm{H}_{2} \mathrm{SO}_{4} / \mathrm{HNO}_{3}$ mixture for $6 \mathrm{~h}$ at $100^{\circ} \mathrm{C}$; inset is the table showing the percentage of each component in the nanotubes.

\subsection{Synthesis of CNT/catalysts nanocomposites}

CNT/metal oxide nanocomposites can be synthesized by different methods which fall into two basic classes. The first class involves the prior synthesis of nanoparticles that subsequently connected to surface functionalized CNTs by either covalent or noncovalent interactions (Eder, 2010; Peng et al., 2010; Hu et al., 2010). The second class is the one step method which involves direct deposition of nanoparticles onto MWCNT surface, in situ formation of nanoparticles through redox reactions or electrochemical deposition on CNTs (Chen et al., 2006; Gavalas et al., 2001; Yang et al., 2010; Sahoo et al., 2001; Lee et al., 2008). The second class has the advantages where uniform nanomaterials can be prepared due to the presence of active sites on oxidized CNT surfaces.

As an example, $\mathrm{CNT} / \mathrm{ZnO}$ nanocomposites are prepared by the following procedure (Saleh et al., 2010). Zinc precursor like $\mathrm{Zn}\left(\mathrm{NO}_{3}\right)_{2} \cdot 6 \mathrm{H}_{2} \mathrm{O}$, is dissolved in doubly deionized water. Then, 
ammonia is added drop-wise under continuous stirring into the solution to form a clear solution. The oxidized MWCNTs is added into the solution. The mixture is refluxed at $100^{\circ} \mathrm{C}$. The composite are separated and dried at $80^{\circ} \mathrm{C}$ prior to the calcination in vacuum at $300^{\circ} \mathrm{C}$.

Different techniques can be applied for the characterization of the nanocomposite. For example XRD is employed to determine crystalline phases and average crystalline size. FT-IR is used for qualitative analysis of the binding of the metal oxide into the nanotube surface. The morphology of the nanotubes and particle size are examined by the field emission scanning electron microscope (FESEM) and high resolution transmission electron microscopy (HRTEM). EDX measurement is also used as a quantitative analysis for the presence of the oxygen containing groups on the surface of the nanotubes. As an example, Figure 5 depicts the EDX data of the CNT/ZnO nanocomposite. The table shows the percentage of each component in the composite. Figure 6, SEM image and the inset HRSEM image, confirm the presence of zinc oxide particles on the surface of the nanotubes.

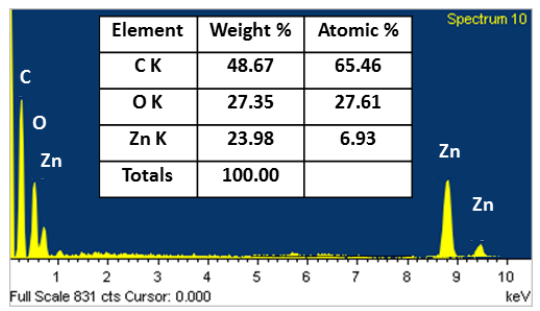

Figure 5. EDX spectrum of the MWCNT/ZnOnanocomposites; inset is the table showing the percentage of each component in the nanotubes.

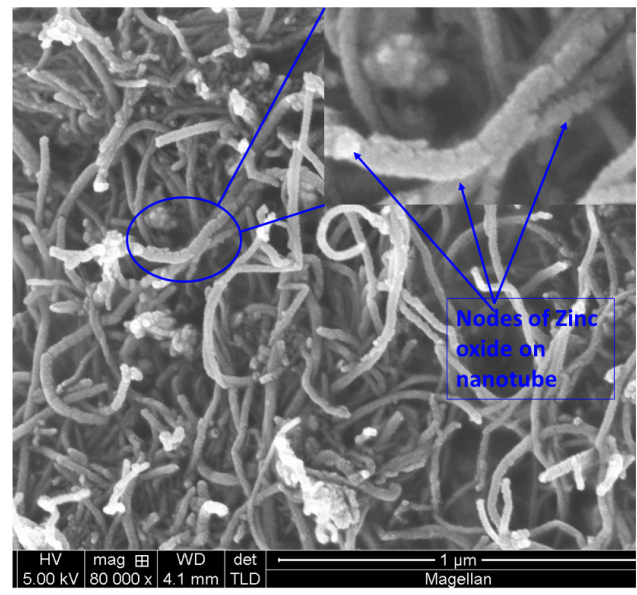

Figure 6. Field emission scanning electron microscopy (FESEM) image of the MWCNT/ZnO; Inset is the HRSEM image. 


\section{Applications of CNT/Catalyst nanocomposites}

CNTs are considered to be good support material for catalysts, because they provide large surface area support and also stabilize charge separation by trapping electrons transferred from metal oxides, thereby hindering charge recombination.

A significant number of papers have been published on the application of CNTs in conjunction with $\mathrm{TiO}_{2}$, reflecting the focus of recent research (Jitianu et al., 2004; Huang and Gao, 2003; Woan et al., 2009; Feng et al., 2005). One of the most important applications of such composite is to act as photocatalyst for some chemical reactions, especially for the decontamination of organic pollutants in waste waters. The photocatalytic activity of $\mathrm{MWCNT} / \mathrm{TiO}_{2}$ composite toward the degradation of acetone under irradiation of UV light was investigated by the detection of the hydroxyl radical $\left({ }^{\circ} \mathrm{OH}\right)$ signals using electron paramagnetic resonance. It has been reported that the agglomerated morphology and the particle size of $\mathrm{TiO}_{2}$ in the composites change in the presence of CNTs, which provide a large surface area resulting in more hydroxyl group on the surface of the composite with no effect on the mesoporous nature of the $\mathrm{TiO}_{2}$. The composite have been reported to be of higher photocatalytic activity than commercial photocatalyst (P25) and $\mathrm{TiO}_{2}$ /activated carbon (AC) composite (Yu et al., 2005a,b).

The photocatalytic activities of $\mathrm{MWCNT} / \mathrm{TiO}_{2}$ under visible light have also been reported using the decolorization of dyes like methylene blue, methyl orange, azo dye and other dyes in model aqueous solutions (Cong et al., 2011; Gao et al., 2009; Hu et al., 2007; Saleh and Gupta, 2012; Yu et al., 2005; Kuo, 2009). $\mathrm{TiO}_{2}$ loading of $12 \%$ was found to result in the highest photoactivity in comparison with $6 \%$ and $15 \%$ loadings. Little $\mathrm{TiO}_{2}$ or excessive nanotubes addition shielded the $\mathrm{TiO}_{2}$ and reduced the UV intensity, due to photon scattering by the nanotubes. However, a high $\mathrm{TiO}_{2}$ content was found to be ineffective in suppressing exciton recombination because of the large distance between the titania and the nanotubes ( $\mathrm{Li}$ et al., 2012). Optimum ratio of titania and nanotubes provides a large surface area support and stabilize charge separation by trapping electrons transferred from $\mathrm{TiO}_{2}$, thereby hindering charge recombination with minimum photon scattering. The composite provides high surface area which is beneficial for photocatalytic activity, as it provides high concentration of target organic substances around sites activated by ultraviolet (UV) radiation.

Also, the activity of $\mathrm{MWCNT} / \mathrm{TiO}_{2}$ composites has been investigated in photodegradation of phenol and photocatalytic oxidation of methanol under irradiation of visible light (Wang et al., 2005; An et al., 2007; Yao et al., 2008; Dechakiatkrai et al., 2007). The catalysts exhibited enhanced photocatalytic activity for degradation of toluene in gas phase under both visible and simulated solar light irradiation compared with that of commercial Degussa P25 (Wu et al., 2009). It exhibited high activity for the photoreduction of $\mathrm{Cr}(\mathrm{VI})$ in water (Xu et al., 2008). Its efficiency was higher compared to a mechanical mixture of $\mathrm{TiO}_{2}$ and $\mathrm{MWCNTs}$. A probable synergistic effect of $\mathrm{TiO}_{2}$ and MWCNTs in a composite $\mathrm{MWCNT} / \mathrm{TiO} \mathrm{O}_{2}$ on the enhancement of visible light performance, have been proposed where MWCNTs act as support, absorbent, photo-generated transfer station and carbon-doping source to narrow the band gap of $\mathrm{TiO}_{2}$. 
The composite has been reported for photoinactivation of E. coli in visible light irradiation (Akhavan et al., 2009). The efficiency of the nanocomposite was high toward photocatalytic hydrogen generation and for the reduction of $\mathrm{CO}_{2}$ with $\mathrm{H}_{2} \mathrm{O}$ (Dai et al., 2009; Xia et al., 2007).

Zinc oxide, a direct wide band gap $(3.37 \mathrm{eV})$ semiconductor with a large excitation binding energy $(60 \mathrm{meV})$, has been investigated as a potential non-toxic photocatalyst used to successfully degrade organic pollutants. Recently, $\mathrm{ZnO}$ nanoparticles have received much attention due to its high photoactivity in several photochemical, UV light response, photoelectron-chemical processes and its low cost production possibility (Wu et al., 2008; Neudeck et al., 2011; Gondal et al., 2010; Drmosh et al., 2010).

Experimental results proved that $\mathrm{CNT} / \mathrm{ZnO}$ nanocomposites display relatively higher photocatalytic activity than $\mathrm{ZnO}$ nanoparticles for the degradation of some dyes like rhodamine B, azo-dyes, methylene blue, methylene orange (Dai et al., 2012; Zhu et al., 2009). The complete removal of azo-dyes such as acid orange, acid bright red, acid light yellow, after selection of optimum operation parameters such as the illumination intensity, catalyst amount, initial dye concentration and the different structures of the dye on the photocatalytic process, can be achieved in relatively short time by using CNT/ZnO composites.

The MWCNT/ZnO nanocomposites exhibits excellent photocatalytic activity toward other pollutants such as acetaldehyde and cyanide in model solutions (Saleh et al., 2011; Saleh et al., 2010). CNTs act as a photogenerated electron acceptor and retard the recombination of photoinduced electron and hole. The adsorption and photocatalytic activity tests indicate that the CNTs serve as both an adsorbent and a visible light photocatalyst. The experimental results show that the photocatalytic activity of the $\mathrm{ZnO} / \mathrm{MWCNT}$ nanocomposites strongly depends on the synthetic route, which is probably due to the difference of surface states resulted from the different preparation processes (Zhang, 2006; Kim and Sigmund, 2002; Jiang and Gao, 2005;Agnihotri et al., 2006).

$\mathrm{CNT} / \mathrm{WO}_{3}$ nanocomposites have been synthesized via different routs (Pietruszka et al., 2005; Wang et al., 2008; Saleh and Gupta, 2011). The utilization of carbon nanotubes to enhance photocatalytic activity of tungsten trioxide has also been investigated. The photocatalytic activities are greatly improved when $\mathrm{CNT} / \mathrm{WO}_{3}$ nanocomposite has been used for the degradation of pollutants such as rhodamine B under ultraviolet lamp or under sunlight. The results showed that photocatalytic activity of the $\mathrm{MWCNT} / \mathrm{WO}_{3}$ composites prepared by chemical process is higher than that prepared by mechanical mixing. The photocatalytic activity is enhanced when $\mathrm{WO}_{3}$ nanoparticles are loaded on the surface of CNTs. The enhanced photocatalytic activity may be ascribed to the effective electron transfer between the nanotubes and the metal nanoparticles.

A possible synergistic effect between the semiconductor nanoparticles and CNTs on the enhancement of photocatalytic activity is proposed in Figure 7. The mechanism is based on the results of the structure characterizations and the enhancement in photocatalytic activity of the prepared composite.

When the catalyst is irradiated by photons, electrons $\left(\mathrm{e}^{-}\right)$are excited from the valence band (VB) to the conduction band (CB) of catalysts or the metal oxide nanoparticles (NP) creating a 
charge vacancy or holes $\left(\mathrm{h}^{+}\right)$, in the VB. Some of the charges quickly recombine without creating efficient photodecomposition of the pollutant. In the case where the composite is applied, the strong interaction between the nanotube and the metal oxide results in a close contact to form a barrier junction which offers an effective route of reducing electron-hole recombination by improving the injection of electrons into the nanotube. Therefore, CNTs acts as a photo-generated electron acceptor to promote interfacial electron transfer process since CNTs are relatively good electron acceptor while the semiconductor is an electron donor under irradiation (Saleh and Gupta, 2011; Riggs et al., 2000; Subramanian et al., 2004; Geng et al., 2008). The adsorbed oxygen molecules on the nanotubes react with the electrons forming very reactive superoxide radical ion $\left(\mathrm{O}^{*}{ }^{-}\right)$which oxidize the target. On the other side, the hole (h $\left.{ }^{+}\right)$oxidize hydroxyl groups to form hydroxyl radical $\left({ }^{\circ} \mathrm{OH}\right)$ which can decompose the target.

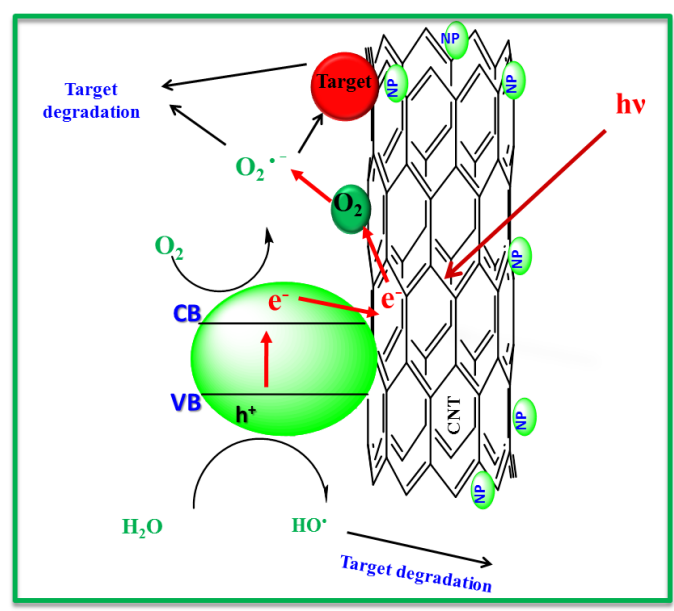

Figure 7. Schematic diagram of the proposed mechanism of photodegradation over CNT/MO composite.

Some important points of such process can be highlighted as:

- Stronger adsorption on photocatalyst for the targeted moleculs of pollutant is achieved by the incorporation of the nanotubes, due to their large specific surface area and high quality active sites.

- The nanotubes can act as effective electron transfer unitbecause of their high electrical conductivity and high electron storage capacity.

- The nanotubes manifest higher capture electron ability and can prompt electron transfer from the conduction band of the metal oxide or semiconductor nanoparticles (NP) towards the nanotube surface due to their lower Fermi level (Cong et al., 2011).

- Schottky barrier forms at the interface between the CNTs and the semiconductor. The photo-generated electrons may move freely towards the CNT surface, thus the left holes may move to the valence band (Woan et al., 2009; Chen et al., 2005). 
- The presence of the nanotubes in the composite can inhibit the recombination of photogenerated electrons and holes, thus, improving the photocatalytic activity.

- The transmission stability of promoted electron between the nanotubes and the conduction band is enhanced by the strong interaction and intimate contact between the nanoparticles and the surface of the nanotubes.

\section{Conclusion}

The chapter discusses the preparation of the nanocomposites consisting of carbon nanotubes and metal oxides like titania, zinc oxide and tungsten trioxide. For the preparation of such composite, the oxygen-containing groups are grafted on the surface of the nanotubes by acid treatment. This is followed by the attachment of the metal oxides nanoparticles on the nanotubes surface. The chapter also highlights the means by which the composite is characterized. These include Fourier transform infrared spectroscope, $X$-ray powder diffraction, field emission scanning electron microscope, energy dispersive $\mathrm{X}$-ray spectroscope and transmission electron microscope.

The UV, visible light and sunlight photocatalytic activity of the CNT-based nanocomposites is higher than that of the metal oxide or mechanical mixture of the metal oxide and CNTs. CNTs are considered to be good support materials for semiconductors like $\mathrm{TiO}_{2}, \mathrm{ZnO}$ and $\mathrm{WO}_{3}$ because nanotubes provide a large surface area support with high quality active sites. Also they stabilize charge separation by trapping electrons, thereby hindering electron-hole recombination by modification of band-gap and sensitization.

\section{Acknowledgements}

The author would like to acknowledge the support of Chemistry Departments, Center of Research Excellence in Nanotechnology \& King Fahd University of Petroleum and Minerals, (KFUPM) Dhahran, Saudi Arabia, for this work.

\section{Author details}

Tawfik A. Saleh*

Address all correspondence to: tawfik@kfupm.edu.sa

Chemistry Department, Center of Excellence in Nanotechnology, King Fahd University of Petroleum \& Minerals, Saudi Arabia 


\section{References}

[1] Agnihotri, S., Mota, J. P. B., Rostam-Abadi, M., \& Rood, M. J. (2006). Adsorption site analysis of impurity embedded single-walled carbon nanotube bundles. Carbon, 44(1), 2376-2383.

[2] Akhavan, O., Abdolahad, M., Abdi, Y., \& Mohajerzadeh, S. (2009). Synthesis of titania/carbon nanotube heterojunction arrays for photoinactivation of E. coli in visible light irradiation. Carbon, 47(14), 3280-3287.

[3] An, G., Ma, W., Sun, Z., Liu, Z., Han, B., Miao, S., Miao, Z., \& Ding, K. (2007). Preparation of titania/carbon nanotube composites using supercritical ethanol and their photocatalytic activity for phenol degradation under visible light irradiation. Carbon, $45,1795-1801$

[4] Burghard, M. (2005). Electronic and vibrational properties of chemically modified singlewall carbon nanotubes. Surf. Sci. Rep., 0167-5729, 58(1-4), 1-109.

[5] Chen, W., Pan, X., Willinger, M. G., Su, D. S., \& Bao, X. (2006). Facile autoreduction of iron oxide/carbon nanotube encapsulates. J. Am. Chem. Soc., 128, 3136-3137.

[6] Chen, Y., Crittenden, J. C., Hackney, S., Sutter, L., \& Hand, H. W. (2005). Preparation of a Novel TiO2-Based p-n Junction Nanotube Photocatalyst. Environ. Sci. Technol., 39(5), 1201-1208.

[7] Cong, Y., Li, X., Qin, Y., Dong, Z., Yuan, G., Cui, Z., \& Lai, X. (2011). Carbon-doped $\mathrm{TiO}_{2}$ coating on multiwalled carbon nanotubes with higher visible light photocatalytic activity. Applied Catalysis B: Environmental, 107, 128-134.

[8] Dai, K., Dawson, G., Yang, S., Chen, Z., \& Lu, L. (2012). Large scale preparing carbon nanotube/zinc oxide hybrid and its application for highly reusable photocatalyst. Chemical Engineering Journal, 191, 571-578.

[9] Dai, K., Peng, T., Ke, D., \& Wei, B. (2009). Photocatalytic hydrogen generation using a nanocomposite of multi-walled carbon nanotubes and $\mathrm{TiO}_{2}$ nanoparticles under visible light irradiation. Nanotechnology, 20(12), 125603.

[10] Dechakiatkrai, C., Chen, J., Lynam, C., Phanichphant, S., \& Wallace, G. G. (2007). Photocatalytic oxidation of methanol using titanium dioxide/single-walled carbon nanotube composite. J Electrochem Soc., 154(5), A407-411.

[11] Dresselhaus, M. S., \& Dresselhaus, G. (2001). Carbon Nanotubes: Synthesis, Structure, Properties and Applications: Topics in Applied Physics, Springer-Verlag. ISBN 3-54041-086-4, Berlin.

[12] Drmosh, Q. A., Gondal, M. A., Yamani, Z. H., \& Saleh, T. A. (2010). Spectroscopic Characterization Approach to Study Surfactants Effect On ZnO Nanoparticles Synthesis by Laser Ablation Process. Applied Surface Science, 256, 4661-4666. 
[13] Ebbesen, T. W., Lezec, H. J., Hiura, H., Bennett, J. W., Ghaemi, H. F., \& Thio, T. (2010). Electrical conductivity of individual carbon nanotubes. Nature, 382, 6586 (1996) 54-56.

[14] Eder, D. Carbon nanotube-inorganic hybrids. Chem. Rev., 110, 1348-1385.

[15] Feng, W., Feng, Y., Wu, Z., Fujii, A., Ozaki, M., \& Yoshino, K. (2005). Optical and electrical characterizations of nanocomposite film of titania adsorbed onto oxidized multiwalled carbon nanotubes. J Phys Condens Matter, 17(27), 4361-4368.

[16] Gao, B., Chen, G. Z., \& Li, P. G. (2009). Carbon nanotubes/titanium dioxide $\left(\mathrm{CNTs} / \mathrm{TiO}_{2}\right)$ nanocomposites prepared by conventional and novel surfactant wrapping sol-gel methods exhibiting enhanced photocatalytic activity. Appl Catal, B89(3-4), 503-509.

[17] Gavalas, V. G., Andrews, R., Bhattacharyya, D., \& Bachas, L. G. (2001). Carbon nanotube sol-gel composite materials. Nano Lett., 1, 719-721.

[18] Geng, Q., Guo, Q., Cao, C., \& Wang, L. (2008). Investigation into $\mathrm{NanoTiO}_{2} / \mathrm{ACSPCR}$ for Decomposition of Aqueous Hydroquinone. Ind. Eng. Chem. Res., 47, 2561-2568.

[19] Gondal, M. A., Drmosh, Q. A., Yamani, Z. H., \& Saleh, T. A. (2010). Effect of postannealing temperature on structural and optical properties of nano- $\mathrm{ZnO}$ synthesized from $\mathrm{ZnO}_{2}$ by Laser Ablation Method. International Journal of NanoParticles, 3(3), 257-266.

[20] Hu, G., Meng, X., Feng, X., Ding, Y., Zhang, S., \& Yang, M. (2007). Anatase $\mathrm{TiO}_{2}$ nanoparticles/carbon nanotubes nanofibers: preparation, characterization and photocatalytic properties. J Mater Sci, 42(17), 7162-7170.

[21] Hu, L., Hecht, D. S., \& Gruner, G. (2010). Carbon nanotube thin films: fabrication, properties, and applications. Chem. Rev., 110, 5790-5844.

[22] Huang, Q., \& Gao, L. (2003). Immobilization of rutile $\mathrm{TiO}_{2}$ on multiwalled carbon nanotubes. J Mater Chem, 13(7), 1517-9.

[23] Iijima, S. (1991). Helical Microtubules of Graphitic Carbon. Nature, 354, 56-58.

[24] Jiang, L., \& Gao, L. (2005). Fabrication and characterization of ZnO-coated multi-walled carbon nanotubes with enhanced photocatalytic activity. Mater. Chem. Phys., 91, 313-316.

[25] Jitianu, A., Cacciaguerra, T., Benoit, R., Delpeux, S., Beguin, F., \& Bonnamy, S. (2004). Synthesis and characterization of carbon nanotubes- $\mathrm{TiO}_{2}$ nanocomposites. Carbon, 42(5-6), 1147-1151.

[26] Kim, H., \& Sigmund, W. (2002). Zinc oxide nanowires on carbon nanotubes. Appl. Phys. Lett., 81, 2085-2088. 
[27] Koci, K., Mateju, K., Obalova, L., Krejcikova, S., Lacny, Z., Placha, D., Capek, L., Hospodkova, A., \& Solcova, O. (2010). Effect of silver doping on the $\mathrm{TiO}_{2}$ for Photocatalytic reduction of $\mathrm{CO}_{2}$. Applied Catalysis B: Environmental, 96, 239-244.

[28] Koci, K., Obalová, L., \& Lacný, Z. (2008). Photocatalytic reduction of $\mathrm{CO}_{2}$ over $\mathrm{TiO}_{2}$ based catalysts. Chemical Papers, 62, 1-9.

[29] Koci, K., Reli, M., Kozák, O., Lacny, Z., Plachá, D., Praus, P., \& Obalov, L. (2011). Influence of reactor geometry on the yield of $\mathrm{CO} 2$ Photocatalytic reduction. Catalysis Today, 176(1), 212-214.

[30] Kuo, C-Y. (2009). Prevenient dye-degradation mechanisms using UV/ $\mathrm{TiO}_{2} /$ carbon nanotubes process. J Hazard Mater, 163(1), 239-244.

[31] Lee, D. H., Park, J. G., Choi, K. J., Choi, H. J., \& Kim, D. W. (2008). Preparation of brookite-Type $\mathrm{TiO}_{2}$ /carbon nanocomposite electrodes for application to $\mathrm{Li}$ ion batteries. Eur. J. Inorg. Chem., 6, 878-882.

[32] Li, Y., Leiyong, Li., Chenwan, Li., Chen, W., \& Zeng, M. (2012). Carbon nanotube/ titania composites prepared by a micro-emulsion method exhibiting improved photocatalytic activity. Applied Catalysis A: General, 427(428), 1-7.

[33] Lu, C., Su, F., \& Hu, S. (2008). Surface modification of carbon nanotubes for enhancing BTEX adsorption from aqueous solutions. Applied Surface Science, 254, 7035-7041.

[34] Meyyappan, M. (2004). (Ed.), Carbon Nanotubes: Science and Applications, CRC Press, 0-84932-111-5.

[35] Neudeck, C., Kim, Y. Y., Ogasawara, W., Shida, Y., Meldrum, F., \& Walsh, D. (2011). General route to functional metal oxide nanosuspensions, enzymatically deshelled nanoparticles, and their application in photocatalytic water splitting. Small, 7, 869-873.

[36] Peng, X., Sfeir, M. Y., Zhang, F., Misewich, J. A., \& Wong, S. S. (2010). Covalent synthesis and optical characterization of double-walled carbon nanotube-nanocrystal heterostructures. J. Phys. Chem. C, 114, 8766-8773.

[37] Pietruszka, B., Gregorio, F. D., Keller, N., \& Keller, V. (2005). High-efficiency WO3/ carbon nanotubes for olefin skeletal isomerization. Catal. Today, 102-103, 94-100.

[38] Riggs, J. E., Guo, Z., Carroll, D. L., \& Sun, Y. P. (2000). Strong Luminescence of Solubilized Carbon Nanotubes. J. Am. Chem. Soc., 122, 5879-5880.

[39] Ros, T. G., van Dillen, A. J., Geus, J. W., \& Koningsberger, D. C. (2002). Surface oxidation of carbon nanofibres. Chem Eur J, 8, 1151-1162.

[40] Rosca, I. D., Watari, F., Uo, M., \& Akasaka, T. (2005). Oxidation of multiwalled carbon nanotubes by nitric acid. Carbon, 43, 3124-31.

[41] Rusu, C. N., \& Yates Jr, J. T. (1997). Defect sites on TiO2(110). Detection by O2 photodesorption. Langmuir, 13(16), 4311-4316. 
[42] Sahoo, S., Husale, S., Karna, S., Nayak, S. K., \& Ajayan, P. M. (2011). Controlled assembly of Ag nanoparticles and carbon nanotube hybrid structures for biosensing. J. Am. Chem. Soc., 133, 4005-4009.

[43] Saleh, T. A. (2011). The influence of treatment temperature on the acidity of MWCNT oxidized by $\mathrm{HNO} 3$ or a mixture of HNO3/H2SO4. Applied Surface Science, 257, 17 June, 7746-7751.

[44] Saleh, T. A., \& Gupta, V. K. (2011). Functionalization of tungsten oxide into MWCNT and its application for sunlight-induced degradation of rhodamine B. Journal of Colloid and Interface Science, 362(2), 337-344.

[45] Saleh, T. A., \& Gupta, Vinod. K. (2012). Photo-catalyzed degradation of hazardous dye methyl orange by use of a composite catalyst consisting of multi-walled carbon nanotubes and titanium dioxide. Journal of Colloid and Interface Science, 371(1), 101-106.

[46] Saleh, T. A., Gondal, M. A., Drmosh, Q. A., Z Yamani, H. A., \& AL-yamani, A. (2011). Enhancement in photocatalytic activity for acetaldehyde removal by embedding ZnO nano particles on multiwall carbon nanotubes. Chemical Engineering Journal, 166(1), 407-412.

[47] Saleh, T. A., Gondal, M. A., \& Drmosh, Q. A. (2010). Preparation of a MWCNT/ZnO nanocomposite and its photocatalytic activity for the removal of cyanide from water using a laser. Nanotechnology, 21(49), 8, doi:10.1088/0957-4484/21/49/495705.

[48] Subramanian, V., Wolf, E., \& Kamat, P. V. (2004). Catalysis with TiO2/Gold Nanocomposites. Effect of Metal Particle Size on the Fermi Level Equilibration. J. AM. CHEM. SOC., 126, 4943-4950.

[49] Wang, S., Xiaoliang, Shi., Gangqin, Shao., Xinglong, Duan., Hua, Yang., \& Tianguo, Wang. (2008). Preparation, characterization and photocatalytic activity of multi-walled carbon nanotube-supported tungsten trioxide composites. Journal of Physics and Chemistry of Solids, 69, 2396-2400.

[50] Wang, W. D., Serp, P., Kalck, P., \& Faria, J. L. (2005). Visible light photodegradation of phenol on $\mathrm{MWCNT}-\mathrm{TiO}_{2}$ composite catalysts prepared by a modified sol-gel method. J Mol Catal A: Chem, 235(1-2), 194-9.

[51] Woan, K., Pyrgiotakis, Georgios., \& Sigmund, Wolfgang. (2009). Photocatalytic Carbon-Nanotube-TiO2 Composites. Advanced Materials, 21(21), 2233-2239.

[52] Wu, X., Jiang, P., Cai, W., Bai, X. D., Gao, P., \& Xie, S. S. (2008). Hierarchical ZnO micro-/nanostructure film. Adv. Eng. Mater., 10, 476-481.

[53] Wu, Z., Fan, Dong., Weirong, Zhao., Haiqiang, Wang., Yue, Liu., \& Baohong, Guan. (2009). The fabrication and characterization of novel carbon doped $\mathrm{TiO}_{2}$ nanotubes, nanowires and nanorods with high visible light photocatalytic activity. Nanotechnolo$g y, 20(23), 235701$. 
[54] Xia, W., Wang, Y., Bergstraberr, R., Kundu, S., \& Muhler, M. (2007). Surface characterization of oxygen-functionalized multi-walled carbon nanotubes by high-resolution X-ray photoelectron spectroscopy and temperature-programmed desorption. Applied Surface Science, 254, 247-250.

[55] Xia, X-H., Jia, Z-J., Yu, Y., Liang, Y., Wang, Z., \& Ma, L-L. (2007). Preparation of multi-walled carbon nanotube supported $\mathrm{TiO}_{2}$ and its photocatalytic activity in the reduction of $\mathrm{CO}_{2}$ with $\mathrm{H}_{2} \mathrm{O}$. Carbon, 45(4), 717-21.

[56] Xu, Z., Long, Y., Kang, S-Z., \& Mu, J. (2008). Application of the composite of $\mathrm{TiO}_{2}$ nanoparticles and carbon nanotubes to the photoreduction of $\mathrm{Cr}(\mathrm{VI})$ in water. J Dispersion Sci Technol, 29(8), 1150-2.

[57] Yang, C., Wohlgenannt, M., Vardeny, Z. V., Blau, W. J., Dalton, A. B., Baughman, R., \& Zakhidov, A. A. (2003). Photoinduced charge transfer in poly(p-phenylene vinylene) derivatives and carbon nanotube/C60 composites. Physica B: Condensed Matter, 338(1-4), 366-369.

[58] Yang, D.-Q., Rochette, J-F., \& Sacher, E. (2005). Functionalization of multiwalled carbon nanotubes by mild aqueous sonication. J Phys Chem B, 109, 7788-7794.

[59] Yang, J., Jiang, L. C., Zhang, W. D., \& Gunasekaran, S. (2010). Highly sensitive nonenzymatic glucose sensor based on a simple two-step electrodeposition of cupric oxide $(\mathrm{CuO})$ nanoparticles onto multi-walled carbon nanotube arrays. Talanta, 82, 25-33.

[60] Yao, Y., Li, G., Ciston, S., Lueptow, R. M., \& Gray, K. A. (2008). Photoreactive $\mathrm{TiO}_{2} /$ carbon nanotube composites: synthesis and reactivity. Environ Sci Technol, 42(13), 4952-7.

[61] Yu, J. C., Zhang, L., \& Yu, J. (2002). Rapid synthesis of mesoporous $\mathrm{TiO}_{2}$ with high photocatalytic activity by ultrasound-induced agglomeration. New Journal of Chemistry, 26(4), 416-420.

[62] Yu, Y., Yu, J. C., Chan, C-Y., Che, Y-K., Zhao, J-C., \& Ding, L. (2005a). Enhancement of adsorption and photocatalytic activity of $\mathrm{TiO} 2$ by using carbon nanotubes for the treatment of azo dye. Appl Catal B, 61(1-2), 1-11.

[63] Yu, Y., Yu, Jimmy C., Yu, Jia-Guo., Kwok, Yuk-Chun., Che, Yan-Ke., Zhao, Jin-Cai., Ding, Lu, Ge, Wei-Kun, \& Wong, Po-Keung. (2005b). Enhancement of photocatalytic activity of mesoporous $\mathrm{TiO} 2$ by using carbon nanotubes. Applied Catalysis A: General, 289, 186-196.

[64] Zhang, W. D. (2006). Growth of ZnO nanowires on modified well-aligned carbon nanotube arrays. Nanotechnology, 17, 1036-1040.

[65] Zhu, L.-P., Liao, Gui-Hong, Huang, Wen-Ya, Ma, Li-Li, Yang, Yang, Yu, Ying, \& Fu, Shao-Yun. (2009). Preparation, characterization and photocatalytic properties of ZnO-coated multi-walled carbon nanotubes. Materials Science and Engineering B, 163, 194-198. 
